3-2013

\title{
An Active Disturbance Rejection Based Approach to Vibration Suppression in Two-Inertia Systems
}

\author{
Shen Zhao \\ Cleveland State University \\ Zhiqiang Gao \\ Cleveland State University, Z.GAO@csuohio.edu
}

Follow this and additional works at: https://engagedscholarship.csuohio.edu/enece_facpub

Part of the Electrical and Computer Engineering Commons

How does access to this work benefit you? Let us know!

\section{Publisher's Statement}

This is the accepted version of the following article: S. Zhao and Z. Gao, "An Active Disturbance Rejection Based Approach to Vibration Suppression in Two-Inertia Systems," Asian Journal of Control, vol. 15, no. 2, pp. 350-362, 2013/03/01 2013. doi:10.1002/asjc.552, which has been published in final form at https://onlinelibrary.wiley.com/doi/10.1002/asjc.552

\section{Repository Citation}

Zhao, Shen and Gao, Zhiqiang, "An Active Disturbance Rejection Based Approach to Vibration Suppression in Two-Inertia Systems" (2013). Electrical Engineering \& Computer Science Faculty Publications. 438.

https://engagedscholarship.csuohio.edu/enece_facpub/438

This Article is brought to you for free and open access by the Electrical Engineering \& Computer Science Department at EngagedScholarship@CSU. It has been accepted for inclusion in Electrical Engineering \& Computer Science Faculty Publications by an authorized administrator of EngagedScholarship@CSU. For more information, please contact library.es@csuohio.edu. 


\title{
AN ACTIVE DISTURBANCE REJECTION BASED APPROACH TO VIBRATION SUPPRESSION IN TWO-INERTIA SYSTEMS
}

\author{
Shen Zhao and Zhiqiang Gao
}

\section{INTRODUCTION}

Vibration suppression is important in motion control applications because vibration causes dynamic stresses, energy wastes and performance degradations [1]. By law of physics, mechanical resonance is unavoidable in every system involving motion, but the natural frequencies of such systems are usually quite high and not excited during most common motion maneuvers, where a simple proportionalintegral-derivative (PID) controller is often sufficient to meet the design requirements. Control design becomes an issue, however, when the performance improvements push the loop bandwidth to its limit where the resonant modes come into play. The most common resonance seen in industry can be attributed to the compliant couplings, such as gear boxes, long shafts and belts, which can be treated as springs [2].

To deal with resonance, there are mechanical and electrical means. Since the resonance is caused by compliance, a stiffer transmission, i.e. a direct coupling in place of a belt, will be an obvious solution. Adding more mechanical damping will surely be helpful. In addition, increasing the motor inertia is found to be an effective way to alleviate the resonance [2]. These mechanical methods are costly, which leads us to electrical options, consist of low-pass filter, notch filter [3] and bi-quad filter [4], all for the objective of attenuating the loop gain amplitude at the resonant frequency so that the resonance is suppressed. Some of the electrical methods are equivalent mathematically 
to the mechanical methods mentioned above. Active resonance damping control [4] actually increases the effective physical damping by adding a torque that is proportional to the speed difference between the motor and load. In [5], the active suspension indeed increases the effective damping or spring constant depends on the control design. Acceleration feedback control $[4,6]$, however, increases the motor inertia equivalently. There are still other control methods available, such as center of mass control $[2,4]$ and resonance ratio control $[7,8]$.

All of the above control methods predicate on the detailed mathematical model of the physical process that may or may not be readily available. Even if such a model is obtained at considerable cost, the parameters of the model often change during operation, which may lead to variations in the resonant frequency, leaving the notch filter approach, for example, vulnerable. The attempt to address this flaw leads to solutions such as the adaptive notch filter [9], which is designed to tune the filter parameters on the fly based on adaptive control theory, adding complexity and cost to the design, implementation, and tuning of the control system. It is in this background that an alternative solution is proposed in this paper.

To deal with the resonance problem in motion control, as described above, we resort to a rather novel control method that requires very little system model information and makes the control system tolerant of unknown changes in system dynamics. This method is known as active disturbance rejection control (ADRC) [10-15], based on the key concept of treating the unknown dynamics and disturbances in a physical process as the total disturbance, building a state observer, known as the extended state observer (ESO), to estimate it in real time, and then canceling its effect using a part of the control signal. In the context of the motion control, the resonant mode is not canceled out using a notch filter, but its effect to motion, the ripples in torque, is estimated and canceled in real time using the motor torque, after which the motion dynamic behaves largely like a rigid body. Note that a similar disturbance rejection method was shown in [16], where only the external disturbance is estimated using a state observer and transfer function combined design based on detailed model information.

This paper is organized as follows. The problem description based on two-inertia system model is given in Section II, followed by the main result in Section III, where the motion control problem is reformulated in the context of ADRC. Simulation results and comparison to existing methods are shown in Section IV. Hardware experiments are conducted to verify the simulation results and are presented in Section V. Finally concluding remarks are included in Section VI.

\section{PROBLEM DESCRIPTION AND EXISTING SOLUTION}

The compliant resonance problem can be simplified and represented by the two-inertia system model $[2,8]$ as shown in Fig. 1.

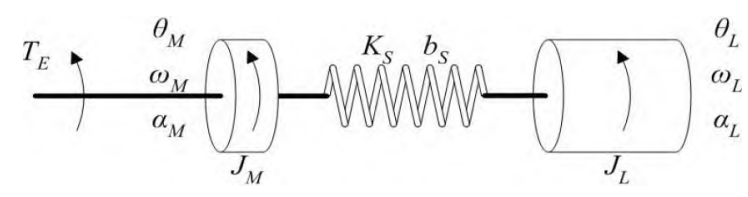

Fig. 1. Two-inertia system model.

Motor inertia $\left(J_{M}\right)$ is connected to load inertia $\left(J_{L}\right)$ by a spring $\left(K_{S}\right)$ with damping $\left(b_{S}\right)$. A torque $\left(T_{E}\right)$ is applied on the motor side to drive the system. Angular acceleration, angular velocity and angular position of the motor and the load are denoted as $\alpha_{M}, \omega_{M}, \theta_{M}$, and $\alpha_{L}, \omega_{l}, \theta_{L}$, respectively. Through simple analysis, we can derive the transfer functions from input $\left(T_{E}\right)$ to different outputs $\left(\omega_{M}, \omega_{L}, \theta_{M}\right.$, and $\left.\theta_{L}\right)$. The transfer function from $T_{E}$ to $\omega_{M}$ is

$$
\frac{\omega_{M}}{T_{E}}=\frac{1}{\left(J_{M}+J_{L}\right) s} \frac{J_{L} s^{2}+b_{S} s+K_{S}}{J_{P} s^{2}+b_{S} s+K_{S}}
$$

where $J_{P}=J_{M} J_{L} /\left(J_{M}+J_{L}\right)$. Similarly, we can get the other three transfer functions.

$$
\begin{aligned}
\frac{\theta_{M}}{T_{E}} & =\frac{1}{\left(J_{M}+J_{L}\right) s^{2}} \frac{J_{L} s^{2}+b_{S} s+K_{S}}{J_{P} s^{2}+b_{S} s+K_{S}} \\
\frac{\omega_{L}}{T_{E}} & =\frac{1}{\left(J_{M}+J_{L}\right) s} \frac{b_{S} s+K_{S}}{J_{P} s^{2}+b_{S} s+K_{S}} \\
\frac{\theta_{L}}{T_{E}} & =\frac{1}{\left(J_{M}+J_{L}\right) s^{2}} \frac{b_{S} s+K_{S}}{J_{P} s^{2}+b_{S} s+K_{S}}
\end{aligned}
$$

The first term of each transfer function is exactly the same as the transfer function for the rigid body model; the second term which contains resonance is introduced by the compliance. In both motor and load transfer functions, the denominators of the resonance term will produce a resonant frequency $\left(\omega_{R}\right)$, and the numerator of the resonance term in motor transfer functions will produce an anti-resonant frequency $\left(\omega_{A R}\right)$ [2]. They can be calculated by following equations.

$$
\omega_{R}=\sqrt{K_{S} / J_{P}}
$$




$$
\omega_{A R}=\sqrt{K_{S} / J_{L}}
$$

The Bode plots of velocity transfer functions of rigid body model and compliant model (two-inertia system model) are shown in Fig. 2 for comparison. At low frequency (below the anti-resonant frequency) the two models behave the same. The motor and load are connected as a whole just like the rigid body. As frequency goes higher, the motor and load become disconnected and behave differently. Around resonant frequency there is a 180 degree phase difference between the motor and load, which to some extent represents the resonance as well.

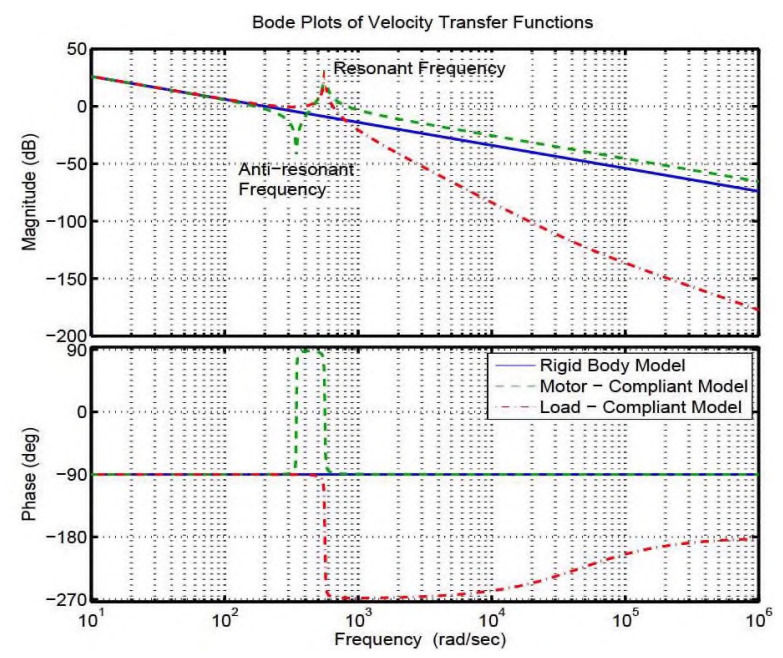

Fig. 2. Bode plots of velocity transfer functions - Rigid vs. Compliant.

Several existing methods are described in [4] that deal with the resonance. A notch filter in the form of

$$
T_{N}(s)=\frac{s^{2}+\omega_{R}^{2}}{s^{2}+2 \zeta \omega_{R} s+\omega_{R}^{2}}
$$

is often used to attenuate the open loop gain at the resonant frequency. The bi-quad filter

$$
T_{B Q}(s)=\frac{s^{2}+2 \zeta_{R} \omega_{R} s+\omega_{R}^{2}}{s^{2}+2 \zeta_{A R} \omega_{A R} s+\omega_{A R}^{2}}
$$

as another solution, not only attenuates the open loop gain at the resonant frequency but also increases the open loop gain at the anti-resonant frequency making it more like a rigid body system. The acceleration feedback method employs a rigid-body Luenberger observer to estimate the motor acceleration and uses it as a feedback for the purpose of increasing the motor inertia.
In a typical configuration of two-inertia system, the sensor is normally mounted at the motor end, where only the motion of the motor is measured and fed back. We denote this set up as motor feedback and this is the common practice in industry. In most cases seen in industry, however, the objective is to control the motion of the load. Consequently, we will also investigate the alternative where we mount the sensor at the load end and use the measurement of the load as feedback, which is denoted as the load feedback. Although the load feedback provides the direct information on how the load behaves, there is a considerable amount of phase lag, comparing to the motor feedback, which makes the control design more challenging. One may suspect that this might be a main reason why the motor feedback configuration is widely used in industry.

Different applications may have different design objectives. Some regulate velocity, others position. To show the generality of the proposed method, both velocity control and position control are addressed in this paper.

\section{The Proposed Solution}

As mentioned in Section $\mathrm{I}$, active disturbance rejection control (ADRC) provides an alternative design paradigm for the resonance problem in motion control. The main idea of ADRC is to treat any unknown dynamics of the system together with external disturbance as a total disturbance, use an extended state observer (ESO) to estimate this total disturbance in real time, and then cancel it in the control law [10]. In this manner we do not have to know the exact system model in order to control it, and particularly in this application we can treat the resonance, no matter what the frequency is, as part of the total disturbance.

For completeness, we consider two types of motion control, velocity control and position control, and two feedback options, motor feedback and load feedback. Since the only difference between velocity control and position control is that the plant has one more integrator in position control, we will only present the problem reformulation for velocity control in the ADRC structure with both feedback options.

\subsection{Velocity control with motor feedback}

With $\quad b_{0}=1 / J_{M}, \quad b_{1}=b_{S} /\left(J_{M} \cdot J_{L}\right), \quad b_{2}=$ $K_{S} /\left(J_{M} J_{L}\right), \quad a_{1}=b_{S} / J_{P}, \quad a_{2}=K_{S} / J_{P}, \quad$ and considering an external disturbance $w$, (1) can be rewritten as

$$
\dddot{y}_{m}+a_{1} \ddot{y}_{m}+a_{2} \dot{y}_{m}=b_{0} \ddot{u}+b_{1} \dot{u}+b_{2} u+w
$$


where $y_{m}$ is the motor velocity, and $u$ is torque applied to the motor. Integrating (9) twice on both sides, the third-order system with a relative degree of one becomes a first-order system [12] as below

$$
\begin{aligned}
\dot{y}_{m}= & b_{0} u+\left(-a_{1} y_{m}-a_{2} \int y_{m}\right. \\
& \left.+b_{1} \int u+b_{2} \iint u+\iint w\right) \\
= & b_{0} u+f\left(y_{m}, \int y_{m}, \int u, \iint u, \iint w\right)(10)
\end{aligned}
$$

Here $f(\cdot)$, including both external disturbance and internal dynamics - the resonance, represents the "total disturbance" to be estimated and mitigated. For the first order system (10), the output $y_{m}$ is defined as the first state $x_{1}$ as usual; additionally, the total disturbance $f$ is defined as the extended state $x_{2}$. Thus the state space representation of $(10)$ is

$$
\left\{\begin{array}{l}
\dot{x}=A_{2} x+b_{0} B_{2} u+E_{2} \dot{f} \\
y_{m}=C_{2} x
\end{array}\right.
$$

where $A_{2}=\left[\begin{array}{ll}0 & 1 \\ 0 & 0\end{array}\right], \quad B_{2}=\left[\begin{array}{l}1 \\ 0\end{array}\right], \quad E_{2}=\left[\begin{array}{l}0 \\ 1\end{array}\right]$, $C_{2}=\left[\begin{array}{ll}1 & 0\end{array}\right]$.

A corresponding second order ESO is then designed as

$$
\dot{z}=A_{2} z+\hat{b}_{0} B_{2} u+L_{2} e_{o}
$$

where $\hat{b}_{0}$ is the estimated value of $b_{0}, L_{2}=$ $\left[\begin{array}{ll}\beta_{1} & \beta_{2}\end{array}\right]^{T}$ is the observer gain vector, and $e_{o}=x_{1}-$ $z_{1}$ is the observer error. The observer gains are selected based on the observer bandwidth defined and discussed in Section 4.1. Mathematical proof has been shown in $[15,17]$ that the observer error is bounded if the derivate of the total disturbance $f$ is bounded, and the bound of the observer error is inversely proportional to the observer bandwidth. With appropriate selection of the observer gains, the observer states $z_{1}$ and $z_{2}$ will track $y_{m}$ and $f$ respectively.

With the total disturbance being estimated, the control law is then designed as

$$
\begin{gathered}
u=\frac{u_{0}-z_{2}}{\hat{b}_{0}} \\
u_{0}=k_{p}\left(r-y_{m}\right)
\end{gathered}
$$

where $k_{l}$, is the controller gain and $r$ is the reference input. Substituting (1.3) into ( $1(1)$,

$$
\dot{y}_{m}=b_{0} \frac{u_{0}-z_{2}}{\hat{b}_{0}}+f \approx u_{0}
$$

Here we can see clearly that the total disturbance is "cancelled" and the plant becomes a pure integrator which can be easily controlled using a proportional controller given in (14).

Compare to the method in [16], the ESO estimates the total disturbance directly, not just the external disturbance, and it uses only a simple, easy to implement and tune state observer, without the need for an additional filter. Furthermore, in the ADRC design less system information is required namely only the motor inertia $J_{M}$, whereas in [16] a full system model is needed.

\subsection{Velocity control with load feedback}

Considering an external disturbance $w,(3)$ can be rewritten as

$$
\dddot{y}_{l}+a_{1} \ddot{y}_{l}+a_{2} \dot{y}_{l}=b_{1} \dot{u}+b_{2} u+w
$$

where $y_{l}$ is the load velocity, and $u$ is torque applied to the motor. Integrating (16) once on both sides, the thirdorder system with a relative degree of two becomes a second-order system

$$
\begin{aligned}
\ddot{y}_{l} & =b_{1} u+\left(-a_{1} \dot{y}_{l}-a_{2} y_{l}+b_{2} \int u+\int w\right) \\
& =b_{1} u+f\left(\dot{y}_{l}, y_{l}, \int u, \int w\right)
\end{aligned}
$$

Similarly, for the second order system (17), define the states $x_{1}=y_{l}, x_{2}=\dot{y}_{l}$ and $x_{3}=f$. The states representation of (17) is

$$
\left\{\begin{array}{l}
\dot{x}=A_{3} x+b_{1} B_{3} u+E_{3} \dot{f} \\
y_{l}=C_{3} x
\end{array}\right.
$$

where $A_{3}=\left[\begin{array}{lll}0 & 1 & 0 \\ 0 & 0 & 1 \\ 0 & 0 & 0\end{array}\right], B_{3}=\left[\begin{array}{lll}0 & 1 & 0\end{array}\right]^{T}, E_{3}=$ $\left[\begin{array}{lll}0 & 0 & 1\end{array}\right]^{T}, C_{3}=\left[\begin{array}{lll}1 & 0 & 0\end{array}\right]$.

A corresponding third order ESO is designed as

$$
\dot{z}=A_{3} z+\hat{b}_{1} B_{3} u+L_{3} e_{o}
$$

where $\hat{b}_{1}$ is the estimated value of $b_{1}, L_{3}=$ $\left[\begin{array}{lll}\beta_{1} & \beta_{2} & \beta_{3}\end{array}\right]^{T}$ is the observer gain vector, and $e_{o}=$ $x_{1}-z_{1}$ is the observer error. With appropriate selection of the observer gains, the observer states $z_{1}, z_{1}$ and $z_{3}$ will track $y_{l}, \dot{y}_{l}$ and $f$ respectively.

The control law is similarly designed as

$$
u=\frac{u_{0}-z_{3}}{\hat{b}_{1}}
$$




$$
u_{0}=k_{p}\left(r-y_{l}\right)+k_{d}\left(\dot{r}-\dot{y}_{l}\right)
$$

where $k_{p}$ and $k_{d}$ are the controller gains. In this case $z_{3}$ is the extended state and a PD controller is designed for the double integrator plant.

For the more detailed derivation of the ADRC control law and recent mathematical analysis of this design approach, the readers are referred to [17-22]. The focus of this paper is on its possible application in motion control in the presence of resonant mode.

\section{SIMULATION RESULTS AND COMPARISON}

In this section, the proposed method is tested in simulation and compared to the three existing methods described in [4], using the motor feedback configuration for velocity control as in [4].

\subsection{Parameters and profile selection}

The proposed method is tested in simulations using the same system parameters as those in [4], with $K_{S}=$ $.372 \mathrm{~N} \cdot \mathrm{m} / \mathrm{rad} . b_{S}=0.008 \mathrm{~N} \cdot \mathrm{m} \cdot \mathrm{s} / \mathrm{rad}, J_{M}=1.88 \times 10^{-3}$ $\mathrm{kg} \cdot \mathrm{m}^{2}, J_{L}=3.13 \times 10^{-3} \mathrm{~kg} \cdot \mathrm{m}^{2}$, and $J_{P}=1.17 \times$ $10^{-3} \mathrm{~kg} \cdot \mathrm{m}^{2}$. In this case, the anti-resonant frequency $\omega_{A R}$ is $345 \mathrm{rad} / \mathrm{s}$ (or $55 \mathrm{~Hz}$ ), and resonant frequency $\omega_{R}$ is $563 \mathrm{rad} / \mathrm{s}$ (or $90 \mathrm{~Hz}$ ). We also compare our method with those discussed in [4] applying their fine tuned parameters in velocity control with motor feedback. The comparison is not done for other cases because [4] only considers velocity control with motor feedback.

Using the parameterization technique proposed in [23], the observer gains and controller gains are selected such that all of the observer eigenvalues are placed at $-\omega_{0}$ and all of the controller eigenvalues are placed at $-\omega_{c}$. Specifically, in a second-order ADRC, $\beta_{1}=$ $2 \omega_{o}, \beta_{2}=\omega_{o}^{2}, k_{p}=\omega_{c}$; in a third-order ADRC. $\beta_{1}=$ $3 \omega_{o}, \beta_{2}=3 \omega_{o}^{2}, \beta_{3}=\omega_{o}^{3}, k_{p}=\omega_{c}^{2}, k_{d}=2 \omega_{c}$; and in a fourth-order ADRC, $\beta_{1}=4 \omega_{o}, \beta_{2}=6 \omega_{o}^{2}, \beta_{3}=4 \omega_{o}^{3}$, $\beta_{4}=\omega_{o}^{4}, k_{p}=\omega_{c}^{3}, k_{d}=3 \omega_{c}^{2}, k_{d d}=3 \omega_{c}$. Above $\omega_{o}$ is the observer bandwidth and $\omega_{c}$ is the controller bandwidth. By fixing the ratio between the observer and controller bandwidth, $\omega_{o}$ becomes the only tuning parameter making the tuning process very easy and intuitive. In this paper we set $\omega_{c}=\omega_{o} / 2$.

Observer and controller bandwidth are selected based on following considerations: 1) the controller bandwidth should be higher than the required bandwidth given in the specification; 2) the observer bandwidth should be two to five times higher than the controller bandwidth; 3) the observer bandwidth should be five to ten times less than the sampling rate. Normally higher the bandwidth is, better the performance is; the cost is that the system is more susceptible to noise and has less robustness.

Step reference is a commonly used profile in simulations and real tests, but it is too aggressive and contains components with very broad bandwidth, which will excite the resonant mode of the system. So in industry the trapezoidal profile, which is less aggressive and also energy saving, is widely used instead of step reference.

Even if a trapezoidal profile is used, the rising time of the profile is still crucial to the system performance. The faster the rising time is, more possible the system is going to have resonance. In order to avoid the resonance, we choose our rising time between $0.05 \mathrm{~s}$ and $0.1 \mathrm{~s}$ in our simulations.

\subsection{Observer performance}

The proposed method is simulated with the rising time set to $100 \mathrm{~ms}(0.1 \mathrm{~s})$, the profile starting time set to $0.5 \mathrm{~s}$ and a disturbance of $1 \mathrm{~N} \cdot \mathrm{m}$ applied to the motor at 1 s. Fig. 3 and Fig. 4 show the plots of the observer states versus the actual system states with the observer bandwidth set to $400 \mathrm{~Hz}$. It is noticed that the error is bounded and converges to zero very quickly indicating very good observer performance.
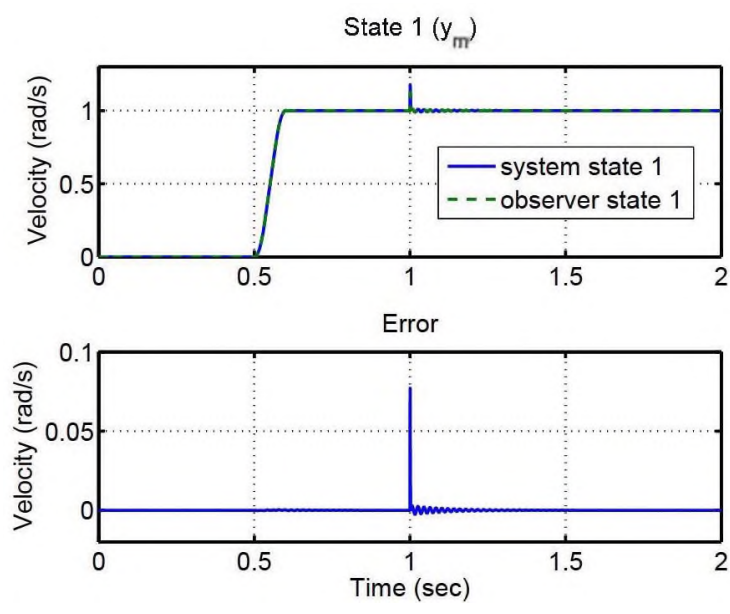

Fig. 3. Estimation of state I.

\subsection{Comparison}

The proposed method is then compared to the notch filter, bi-quad filter, acceleration feedback methods with the same profile and disturbance as 

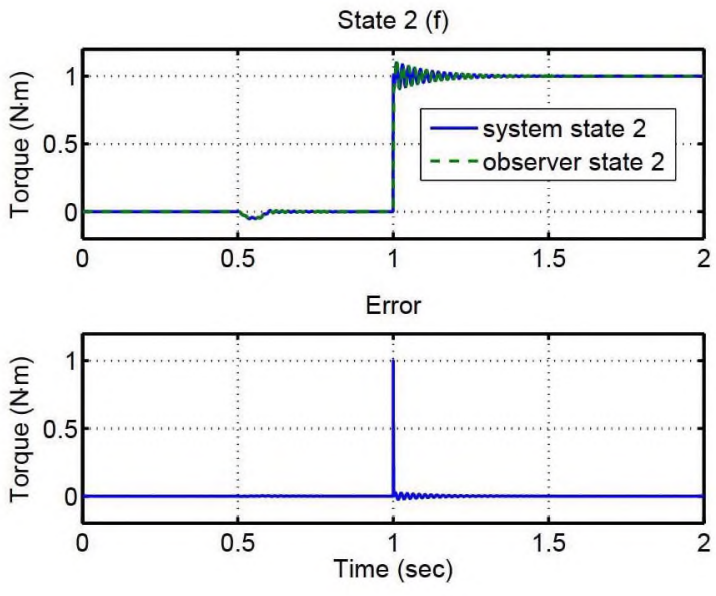

Fig. 4. Estimation of state 2.

Table 1. Motor responses : tracking performance

\begin{tabular}{|c|c|c|c|}
\hline \multicolumn{2}{|c|}{} & $\begin{array}{c}\text { Overshoot } \\
(\%)\end{array}$ & $\begin{array}{c}5 \% \text { Settling Time } \\
(\mathrm{ms})\end{array}$ \\
\hline \multicolumn{2}{|c|}{ Notch Filter } & 4.2 & 133 \\
\hline \multicolumn{2}{|c|}{ Bi-quad Filter } & 1.3 & 115 \\
\hline \multicolumn{2}{|c|}{ Accel. Feedback } & 4.8 & 137 \\
\hline \multirow{2}{*}{\begin{tabular}{c} 
ADRC \\
\multirow{2}{*}{$\omega_{t}(\mathrm{~Hz})$}
\end{tabular}} & 100 & 0.6 & 108 \\
\cline { 2 - 4 } & 200 & 0.2 & 97 \\
\cline { 2 - 4 } & 400 & 0.1 & 96 \\
\hline
\end{tabular}

Table 2. Motor responses : disturbance rejection performance

\begin{tabular}{|c|c|c|c|}
\hline \multicolumn{2}{|c|}{} & $\begin{array}{c}\text { Max. Error } \\
(\%)\end{array}$ & $\begin{array}{c}5 \% \text { Settling Time } \\
(\mathrm{ms})\end{array}$ \\
\hline \multicolumn{2}{|c|}{ Notch Filter } & 135 & $>1000$ \\
\hline \multicolumn{2}{|c|}{ Bi-quad Filter } & 70 & $>1000$ \\
\hline \multicolumn{2}{|c|}{ Accel. Feedback } & 72 & 72 \\
\hline \multirow{2}{*}{$\begin{array}{c}\text { ADRC } \\
\omega_{0}(\mathrm{~Hz})\end{array}$} & 100 & 58 & 66 \\
\cline { 2 - 4 } & 200 & 34 & 86 \\
\cline { 2 - 4 } & 400 & 18 & 94 \\
\hline
\end{tabular}

described in the previous subsection. The results are shown in Table 1 and 2, as well as in Fig. 5.

It is observed that acceleration feedback has the biggest overshoot. Bi-quad filter has less overshoot because it cancels out both resonant and anti-resonant terms in the transfer function. ADRC has even less overshoot and the overshoot decreases as the bandwidth increases. The disturbance rejection ability of acceleration feedback is better than both notch filter and bi-quad filter, which have big errors and oscillate. But ADRC has the best disturbance rejection ability which increases as the bandwidth increases.
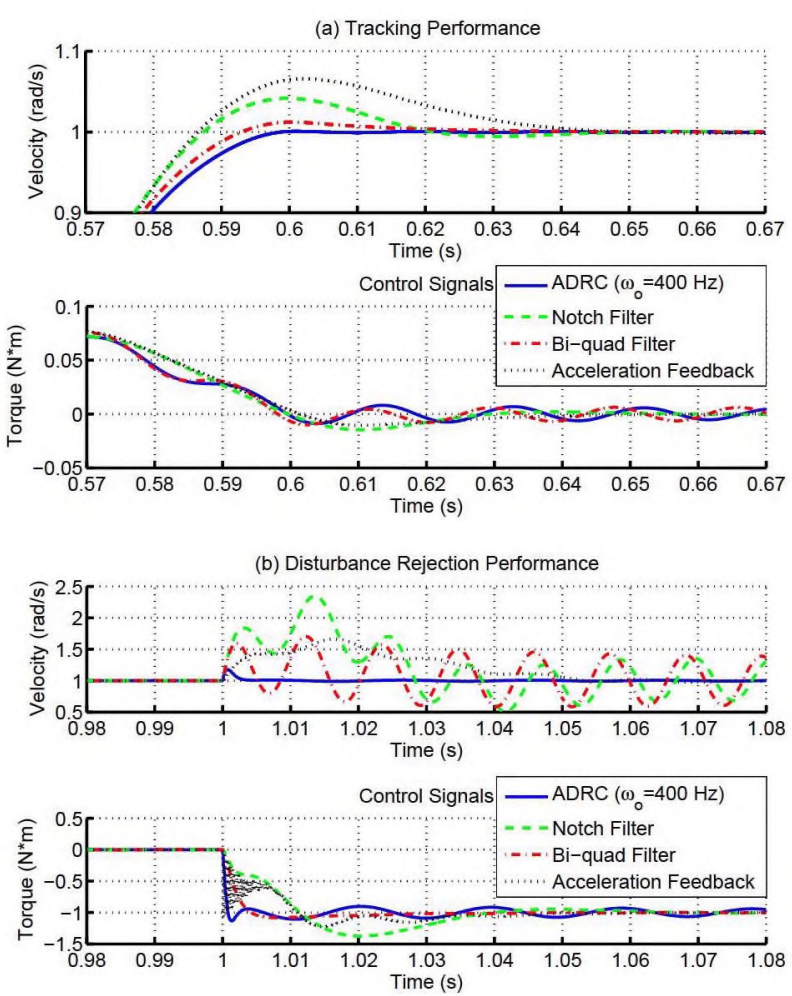

Fig. 5. Motor response comparison. (a) tracking response; (b) disturbance response.

Note that the bandwidth of ADRC can go well beyond the resonant frequency, which is quite difficult to achieve with other methods. As shown in [4] the closed-loop bandwidths associated with the notch filter, the bi-quad filter and acceleration feedback design are $32 \mathrm{~Hz}, 47 \mathrm{~Hz}$ and $37 \mathrm{~Hz}$, respectively, well below the resonant frequency $(90 \mathrm{~Hz})$. Based on the frequency response analysis of ADRC [24], with $\omega_{o}$ set to 400 $\mathrm{Hz}$, however, the closed-loop bandwidth of ADRC is found to be $192 \mathrm{~Hz}$, which is well beyond the resonant frequency, unlike the existing methods.

The robustness of each controller is also tested by varying the load inertia without changing the controller parameters. The tests are performed with the load changing to $0.9,1.1,2$ and 5 times of its original value. The bi-quad filter is found to be the most fragile, because the system becomes unstable for all four load changes. With the notch filter, the system is stable for the first two changes but becomes unstable for last two in the presence of external disturbances. Acceleration feedback and ADRC are stable for all four cases, but the former results in a bigger overshoot of $15 \%$. The motor overshoot in ADRC remains mostly unchanged, 
but the load oscillation becomes more pronounced with the increasing load.

\subsection{Position control}

In this subsection, some simulation results are provided to demonstrate the proposed method works for position control as well. The results are summarized in Table 3. The best performance is obtained at the medium bandwidth of $80 \mathrm{~Hz}$; when the bandwidth goes beyond $150 \mathrm{~Hz}$ the system becomes unstable. Fig. 6 shows the response with $\omega_{o}=80 \mathrm{~Hz}$.

Table 3. Tracking performance of position control

\begin{tabular}{|c|c|c|}
\hline $\begin{array}{c}\omega_{o} \\
(\mathrm{~Hz})\end{array}$ & $\begin{array}{c}\text { Overshoot } \\
(\%)\end{array}$ & $\begin{array}{c}5 \% \text { Settling Time } \\
(\mathrm{ms})\end{array}$ \\
\hline 40 & 0.4 & 157 \\
\hline 60 & 0.3 & 133 \\
\hline 80 & 0.1 & 116 \\
\hline 100 & 0.2 & 105 \\
\hline 120 & 0.3 & 102 \\
\hline
\end{tabular}

(a) Tracking Performance

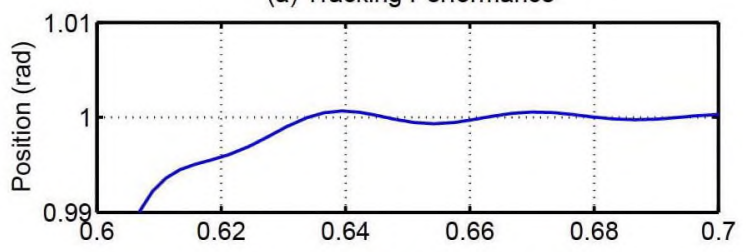

(b) Disturbance Rejection Performance

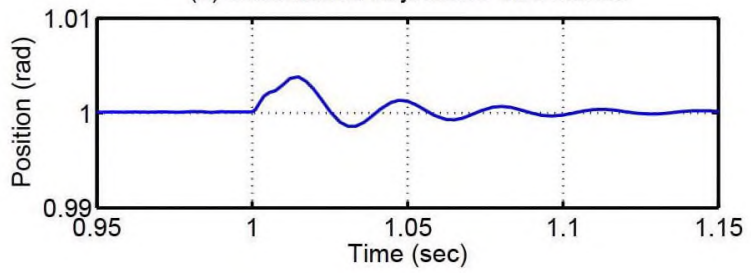

Fig. 6. Position control response. (a) tracking response; (b) disturbance response.

\section{EXPERIMENT VERIFICATION}

In addition to the simulation comparison with other methods, the proposed control solution to the vibration problem is also verified in hardware tests for the velocity control with motor feedback case. The experiments are conducted on the torsional apparatus Model 205 from Educational Control Products. For a fast validation, the control algorithm is implemented using the MATLAB real-time workshop in this paper. For application purpose, the implementation of the proposed algorithm can be found in [25].

\subsection{Test setup}

The torsional apparatus Model 205 has a flexible vertical shaft connecting three disks (lower, middle and upper), with an encoder mounted on the lower disk for the purpose of position measurement. The lower disk is driven by a DC servo motor via belt and pulley system with 3 to 1 speed reduction ratio. In this experiment since we only consider the vibration in a two-inertia system, the upper disk is not used and the belt is tightened to provide a rigid connection that matches the simulation model. There are also brass weights that can be added to the middle disk to test the effect of changing the inertia of the load.

A personal computer (PC), with MATLAB realtime workshop installed, is used to implement the proposed control algorithm. A four-channel quadrature encoder input card (PCI-QUAD04) and a multifunction analog and digital I/O card (PCI-DAS 1002), both from Measurement Computing, are install in the computer to interface with the torsional apparatus. A photo of the experimental system is shown in Fig. 7. A diagram is also given (see Fig. 8) to clearly show the mechanical and electrical connections of the system.

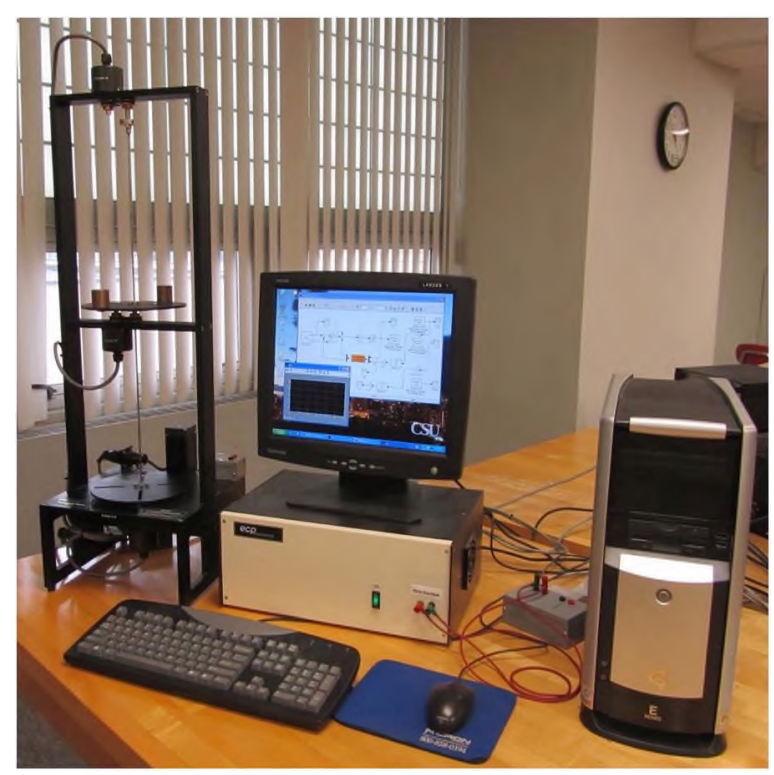

Fig. 7. Photo of the test setup. 


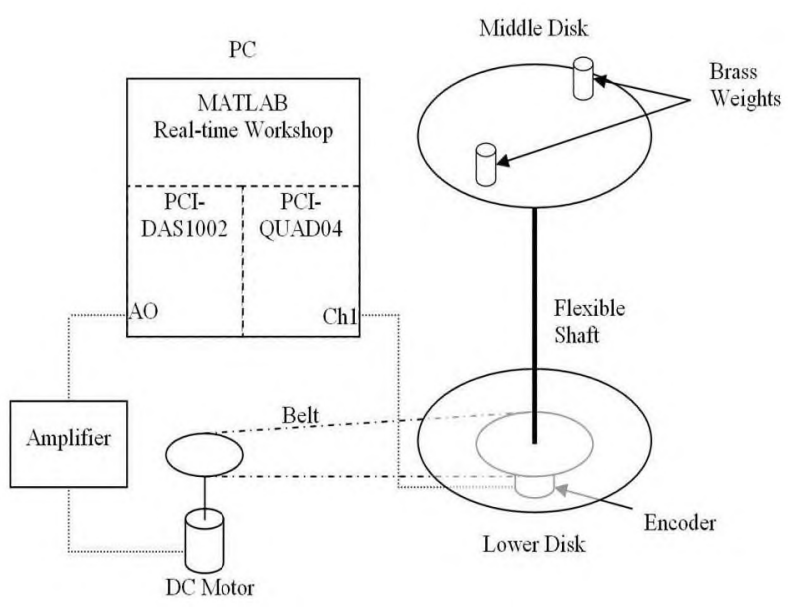

Fig. 8. Diagram of the test setup.

\subsection{System parameters}

The torque constant $\left(K_{T}=T_{E} / U\right)$ of the motor is $0.058 \mathrm{~N} \cdot \mathrm{m} / \mathrm{V}$. The encoders generate 16000 pulses per round. Therefore the resolution for position measurement is $3.927 \times 10^{-4} \mathrm{rad}\left(6.25 \times 10^{-5}\right.$ round). The resolution for velocity measurement depends on the sampling rate, and is $0.196 \mathrm{rad} / \mathrm{s}(0.03125 \mathrm{round} / \mathrm{s})$ at $500 \mathrm{~Hz}$ and $0.393 \mathrm{rad} / \mathrm{s}(0.0625 \mathrm{round} / \mathrm{s})$ at $1 \mathrm{KHz}$, i.e. higher the sampling rate lower the resolution. To get a better resolution, a sampling rate of $500 \mathrm{~Hz}$ is adopted for velocity control.

To determine the parameters of the test equipment, a frequency sweep test is run by applying a chirp signal with an amplitude of 2 volts to the amplifier. The frequency changes from $0.1 \mathrm{~Hz}$ to $15 \mathrm{~Hz}$ in 30 seconds. Fig. 9 shows the motor velocity response. The anti-resonant frequency $\left(\omega_{A R}\right)$ and the resonant frequency $\left(\omega_{R}\right)$ are observed at $37.6 \mathrm{rad} / \mathrm{s}$ (or $5.99 \mathrm{~Hz}$ ) and $48.1 \mathrm{rad} / \mathrm{s}$ (or $7.65 \mathrm{~Hz}$ ) respectively from the test. The peak velocity at the resonant frequency is 3.08 round/s.

From Fig. 2 we can see that at low frequency the motor response and the load response are consistent and the whole system behaves like a rigid body. Thus another test is run with a $0.3 \mathrm{~Hz}$ sinusoid input to determine the total inertia $\left(J_{T}=J_{M}+J_{L}\right)$ of the system. The gain at $0.3 \mathrm{~Hz}$ is found to be $107.76 \mathrm{round} / \mathrm{s} / \mathrm{N} / \mathrm{m}$. From (1) $J_{T}$ is calculated to be $4.92 \times 10^{-3} \mathrm{~kg} \cdot \mathrm{m}^{2}$. Together with the above frequency sweep test results, from (5) and (6), we get $J_{M}=$ $3.01 \times 10^{-3} \mathrm{~kg} \cdot \mathrm{m}^{2}, J_{L}=1.91 \times 10^{-3} \mathrm{~kg} \cdot \mathrm{m}^{2}, J_{P}=$ $1.17 \times 10^{-3} \mathrm{~kg} \cdot \mathrm{m}^{2}, K_{S}=2.71 \mathrm{~N} \cdot \mathrm{m} / \mathrm{rad}, b_{S}=0.006$ $\mathrm{N} \cdot \mathrm{m} \cdot \mathrm{s} / \mathrm{rad}$.

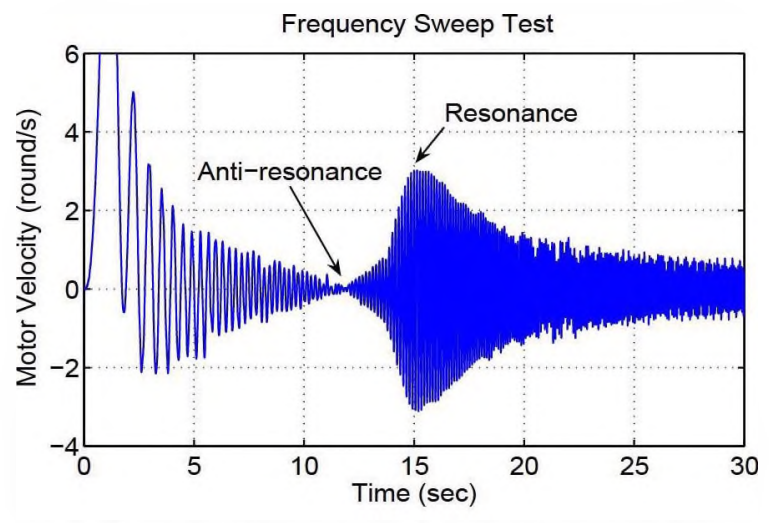

Fig. 9. Motor velocity response of the frequency sweep test.

According to the equipment manual the motor inertia, which includes the inertial of the DC motor, pulley and the lower disk, is around $2.65 \times 10^{-3} \mathrm{~kg} \cdot \mathrm{m}^{2}$ and the load inertia is around $2.00 \times 10^{-3} \mathrm{~kg} \cdot \mathrm{m}^{2}$, which matches the tests quite well.

\subsection{Test results}

A trapezoidal profile, as mentioned in subsection 4.1 , with a magnitude of $8 \mathrm{round} / \mathrm{s}$ is used to run the tests. The rising time is chosen to be 0.5 seconds which is slower, due to a relative lower resonant frequency compared to the simulation case. The controller under test is described in subsection 3.1, with the observer bandwidth and the controller bandwidth set to $320 \mathrm{rad} / \mathrm{s}$ and $160 \mathrm{rad} / \mathrm{s}$ respectively. The results are shown in Fig. 10.

Both motor response and load response track the reference very well before the load change. A load with inertia of $3.29 \times 10^{-3} \mathrm{~kg} \cdot \mathrm{m}^{2}$ is added to the middle disk, which is equivalent to 2.7 times load change, to test the robustness of the control method. The motor velocity remains well controlled with the load change. But the load exhibits oscillations as expected, since resonant frequency is lowered with the load increase and the previous profile is a little fast compare to the new resonance. Test results show that decreasing the rising time to one second will greatly reduce the oscillations.

\subsection{Frequency response analysis}

Based on the system model, the open loop and closed-loop transfer functions are derived using the above system and controller parameters and the Bode plots are given in Fig. 11 and 12. From Fig. 11, the phase margin of the system is found to be 50 degrees. The closed-loop bandwidth is read from Fig. 12 to be 

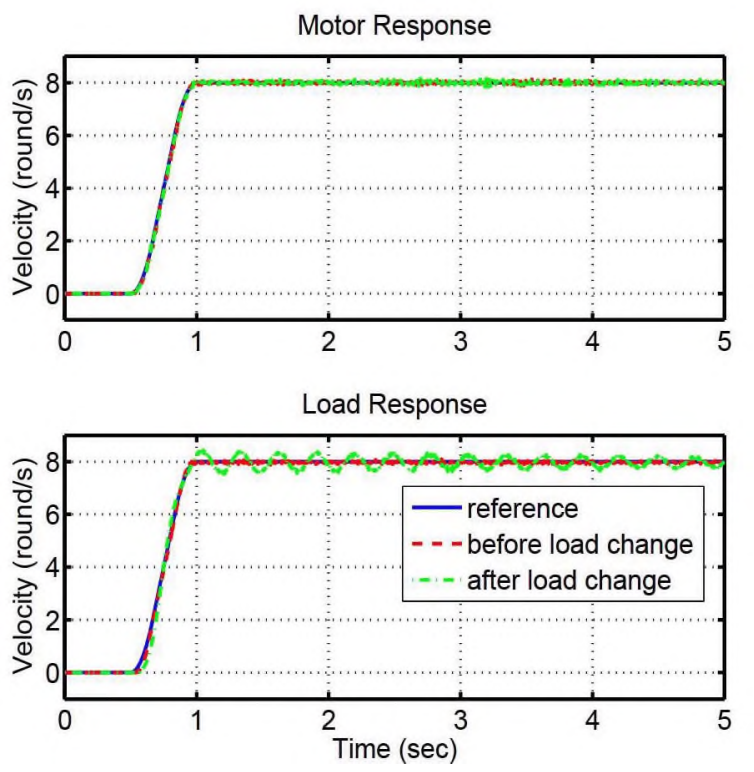

Fig. 10. Velocity control test results.

$158 \mathrm{rad} / \mathrm{s}$, which is well beyond the resonant frequency of the system $(48.1 \mathrm{rad} / \mathrm{s})$. The resonant mode of the system is attenuated by applying the proposed control method.

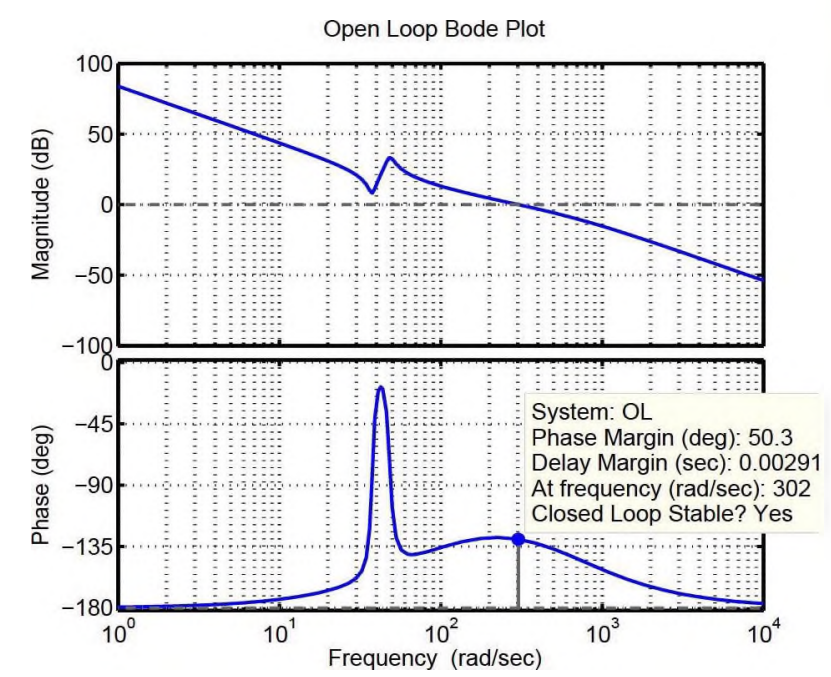

Fig. 11. Open loop Bode plot.

\section{CONCLUSIONS}

A novel solution for resonance suppression in motion control is proposed. By reformulating the

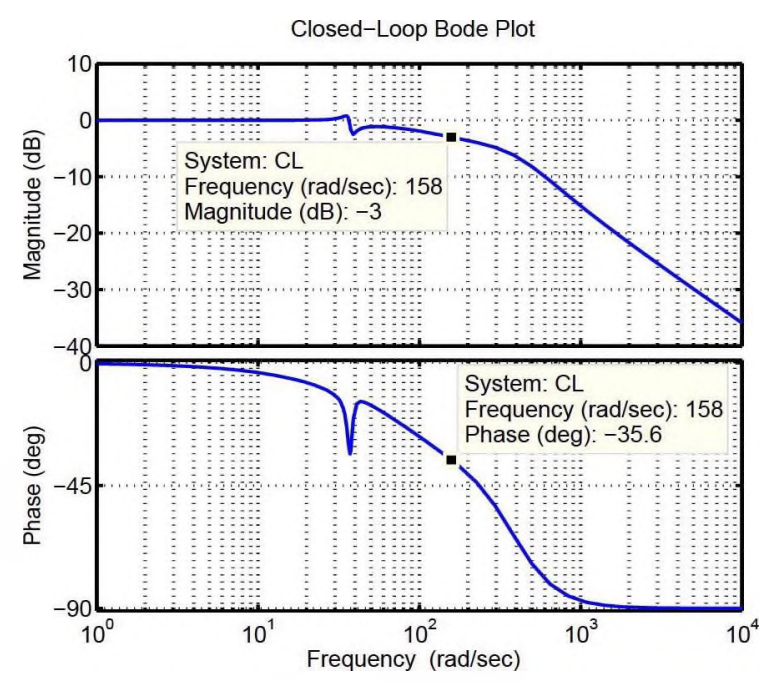

Fig. 12. Closed-loop Bode plot.

problem in the framework of active disturbance rejection control, solutions for both velocity control and position control are presented and compared with the existing methods favorably. It is shown that, with the proposed method, vibration can be eliminated even when the control bandwidth is pushed well beyond the resonant frequency, which is assumed unknown. Both simulation and hardware test results show that the proposed solution works quite well, making it a rather robust and practical solution for motion control.

\section{REFERENCES}

[1] Beards, C., Vibration Analysis and Control System Dynamics, Halsted Press (1981).

[2] Ellis, G., Control System Design Guide, Second Edition, Academic Press, (2000).

[3] Schmidt, P. and T. Rehm, "Notch filter tuning for resonant frequency reduction in dual inertia systems," Proceedings of IEEE Industry Applications Conference, Vol. 3, pp. 1730-1734, (1999).

[4] Ellis, G. and R. D. Lorenz, "Resonant load control methods for industrial servo drives," Proceedings of IEEE Industry Applications Conference, Vol. 3, pp. 1438-1445, (2000).

[5] Gảspár, P., Z. Szabó, G. Szederkényi, and J. Bokor, "Design of a two-level controller for an active suspension system," Asian Journal of Control, doi: 10.1002/asjc.386, (2011). 
[6] Ellis, G., "Cures for mechanical resonance in industrial servo systems," PCIM Conference, pp. 187-192, (2001).

[7] Hori, Y., "2-mass system control based on resonance ratio control and manabe polynomials," Asia Control Conference, pp. 741-744, (1994).

[8] Hori, Y., H. Sawada, and Y. Chun, "Slow resonance ratio control for vibration suppression and disturbance rejection in torsional system," IEEE Transactions on Industrial Electronics, Vol. 46, No. 1, pp. 162-168, (1999).

[9] Zhou, Y., F. Peng, and B. Li, "Adaptive notch filter control for the torsion vibration in lead-screw feed drive system based on neural network," Intelligent Robotics and Applications, Vol. 5315, pp. 803812, (2008).

[10] Gao, Z., Y. Huang, and J. Han, "An alternative paradigm for control system design," Proceedings of the 40th IEEE Conference on Decision and Control, Vol. 5, pp. 4578-4585, (2001).

[11] Gao, Z., "Active disturbance rejection control: a paradigm shift in feedback control system design," American Control Conference, pp. 2399-2405, (2006).

[12] Han, J., "From pid to active disturbance rejection control," IEEE Transactions on Industrial Electronics, Vol. 56, No. 3, pp. 900-906, (2009).

[13] Zheng, Q., L. Dong, D. Lee, and Z. Gao, "Active Disturbance Rejection Control for MEMS Gyroscopes," IEEE Transactions on Control System Technology, Vol. 17, No. 6, pp. 14321438, (2009).

[14] Xia, Y., B. Liu, and M. Fu, "Active disturbance rejection control for power plant with a single loop," Asian Journal of Control, doi: 10.1002/asjc.240, (2011).

[15] Zheng, Q., Z. Chen, and Z. Gao, "A practical approach to disturbance decoupling control," Control Engineering Practice, Vol. 17, pp. 10161025, (2009).

[16] She, J., M. Fang, Y. Ohyama, H. Hashimoto, and $\mathrm{M}$. Wu, "Improving disturbance-rejection performance based on an equivalent-input-disturbance approach," IEEE Transactions on Industrial Electronics, Vol. 55, No. 1, pp. 380-389, (2008).
[17] Zheng, Q., L. Q. Gao, and Z. Gao, "On validation of extended state observer through analysis and experimentation," ASME Journal of Dynamic Systems, Measurement and Control, Vol. 134, No. 2, pp. 024505-6, (2012).

[18] Guo, B.-Z. and Z.-L. Zhao, "On the convergence of an extended state observer for nonlinear systems with uncertainty," Systems \& Control Letters, Vol. 60, No. 6, pp. 420-430, (2011).

[19] Xue, W. and Y. Huang, "Comparison of the DOB Based Control, A Special Kind of PID Control and ADRC," American Control Conference, pp. 43734379, (2011).

[20] Huang, Y., W. Xue, and C. Zhao, "Active disturbance rejection control: methodology and theoretical analysis," Journal of Systems Science and Mathematical Sciences, Vol. 31, No. 9, pp. 1111-1129, (2011). (in Chinese).

[21] Zhou, W., S. Shao, and Z. Gao, "A stability study of the active disturbance rejection control problem by a singular perturbation approach," Applied Mathematical Sciences, Vol. 3, No. 10, pp. 491508, (2009).

[22] Freidovich, L. B. and H. K. Khalil, "Performance recovery of feedback-linearizationbased designs," IEEE Transactions on Automatic Control, Vol. 53, No. 10, pp. 2324-2334, (2008).

[23] Gao, Z., "Scaling and bandwidth-parameterization based controller tuning," American Control Conference, pp. 4989-4996, (2003).

[24] Tian, G. and Z. Gao, "Frequency response analysis of active disturbance rejection based control system," IEEE International Conference on Control Applications, pp. 1595-1599, (2007).

[25] Miklosovic, R., A. Radke, and Z. Gao, "Discrete implementation and generalization of the extended state observer," American Control Conference, pp. 2209-2214, (2006). 